This is the accepted manuscript version of an article accepted for publication in Personality and Individual Differences following peer review. The version of record, S. Hiskey, R. Ayres, L. Andres and N. Troop, 'Support for the location of negative posttraumatic cognitions in the diagnosis of posttraumatic stress disorder', Personality and Individual Differences, Vol 74, pp. 192-195, first published online November 11, 2014, is available online via doi: https://doi.org/10.1016/j.paid.2014.10.021

Published by Elsevier. All rights reserved. 
Cite as: Hiskey, S., Ayres, R., Andrews, L., \& Troop, N.A. (2015). Support for the location of negative posttraumatic cognitions in the diagnosis of posttraumatic stress disorder. Personality and Individual Differences, 74, 192-195.

\section{Support for the location of negative posttraumatic cognitions in the diagnosis of Posttraumatic Stress Disorder}

Hiskey, S., Ayres, R., Andrews, L., \& Troop, N.A. 


\begin{abstract}
:
Objective: To investigate the extent to which negative posttraumatic cognitions predict, and so can be best located within, the DSM-5 (APA, 2013) individual symptom clusters of posttraumatic stress disorder (PTSD). Method: An online survey of traumatic experiences, featuring 528 adults. Results: Negative posttraumatic cognitions seem best placed within the Numbing/Detachment symptom cluster. Negative posttraumatic cognitions relating to the self predict higher levels of symptoms across the clusters. While negative cognitions relating to the world contributed to most symptom groups, self-blame cognitions did not. Conclusions: Our findings support the placement of posttraumatic cognitions as part of the Numbing/Detachment symptom cluster within the DSM-5 rather than the Intrusion, Avoidance and Hyperarousal clusters.
\end{abstract}

Keywords: Posttraumatic Stress Disorder; Posttraumatic Cognitions, Adulthood (18 yrs \& older); DSM-5 


\section{Introduction:}

The latest evolution of the American Psychiatric Association's Diagnostic and Statistical Manual (DSM-5: APA, 2013) has led to changes in the way that posttraumatic stress disorder (PTSD) is conceptualised. For instance what constitutes a traumatic event is now more specific and includes sexual assault as well as recurring exposure, such as that experienced by police officers or regular first responders. In addition, the DSM-5 has increased the number of symptom clusters from three (Intrusion, Avoidance, Hyperarousal) to four (Intrusion, Avoidance, Hyperarousal and Negative alterations in Cognition and Mood). The DSM-IV Avoidance cluster has now been split to feature avoidance of distressing thoughts, feelings or external reminders of the event on one hand and estrangement, diminished interest in activities, numbing and negative cognitions (such as persistent negative beliefs and expectations about oneself or the world, or persistent distorted blame of self or other people for causing the trauma or its sequelae) and mood on the other. In essence negative cognitions, feeling states and numbing in its various guises are now grouped together and are considered separate from active avoidance.

The conceptual shift to feature event-specific appraisals within the core symptom profile of PTSD is perhaps unsurprising given a highly influential cognitive model to explain the maintenance of the disorder (Ehlers \& Clark, 2000). Furthermore, treatments arising from this model have in many ways become a mainstay of clinical intervention (National Institute for Clinical Excellence: NICE, 2005). Indeed a large body of literature has already examined the relationship between negative posttraumatic appraisals and PTSD and has consistently demonstrated strong positive associations between negative cognitive content and symptom severity, both cross-sectionally (e.g. Ehring et al., 2006) and longitudinally (e.g. Ehlers et al., 1998). Further, negative posttraumatic cognitions have 
been shown to account for up to 58\% of the variance in PTSD severity in some regression analyses (Carek et al. 2010).

Thus, that negative cognitions should now feature as a necessary facet of the disorder may not be contentious. However, their location within the specific Numbing/Detachment cluster may be more open to question. To our knowledge there has been no empirical support for the appropriateness of this particular decision, beyond a review by Friedman et al. (2011) that suggested this might be the case. Accurately detailing where to place posttraumatic cognitions, in terms of existing symptom clusters, may be relevant not only to researchers and academics in relation to modelling responses to traumatic events but also to clinicians who are called upon to determine symptom levels to inform treatment and who may also be asked to undertake medico-legal work to define diagnoses and help consider suitable levels of compensation.

Given the above, we sought to explore the extent to which common negative posttraumatic appraisals could predict each of the individual DSM-5 symptom clusters, thereby determining their most appropriate location. In this way we aim to help further delineate the relative structure of posttraumatic symptoms and test empirically the recent changes to a widely used diagnostic system. Given the limited research in this area (other than that cited above) we have no a priori hypothesis.

\section{Method:}

\subsection{Procedure:}

The data for the current study were collected through internet survey methodology which is recognised as an effective and highly accessible means through which to gather data from hard to 
reach populations (Smith \& Leigh, 1997). We utilised a targeted recruitment approach (Buchanan \& Smith, 1999) that allowed those searching the Internet for websites or resources regarding traumatic experiences to find links to the research. A single page outlined the details of the study and included details regarding the aims of the research and the procedure so that potential participants could make a fully informed decision regarding its requirements. Participants were also informed of their right to withdraw from the study at any point should they wish to do so, and information was provided regarding online resources for those who had experienced a traumatic event. The information and questionnaires were presented to the participants in the following order: information sheet, questions pertaining to demographics, the Posttraumatic Diagnostic Scale (PDS; Foa, 1995), and the Posttraumatic Cognitions Inventory (PTCI: Foa et al., 1999). Ethical approval for the project was granted by the University of Essex.

\subsection{Participants:}

Data are reported for 528 participants who met Criterion A for PTSD (DSM-5: APA, 2013). Those taking part were predominately female (81\%) with an average age of $30 \mathrm{yrs}(\mathrm{s} . \mathrm{d}=10 \mathrm{yrs})$. The majority described their ethnicity as White or Caucasian (72\%), with reports of educational attainment suggesting a highly educated sample (25.6\% had attended University and a further $31.8 \%$ held a postgraduate/professional degrees). The nature of the traumatic event the participant had experienced was established via self-report using the PDS. Traumatic life experiences reported included accident/disaster (25\%), non-sexual assault (26\%) and sexual assault (49\%).

\subsection{Measures:}

Posttraumatic Diagnostic Scale (PDS): The PDS is a valid and reliable (Foa et al., 1997) 49-item self-report questionnaire which assesses criteria for PTSD as outlined in the DSM-IV (APA, 1994). 
It measures the severity of symptoms via 17 questions, each rated on a four point Likert scale from 0 (not at all/only once) to 3 (five or more times a week/almost always) over the past month. Five items (e.g. having bad dreams or nightmares about the event) make up the Intrusion cluster, seven make up the Avoidance cluster (e.g. trying not to think about, talk about or have feelings about the event) and five make up the Hyperarousal cluster (e.g. being overly alert). Total symptom scores (PDS-Total) can also be calculated. Furthermore, the DSM-IV Avoidance scale can be split into Avoidance and Numbing/Detachment scales which is more in line with DSM-5 symptom groupings (see Table One).

Posttraumatic Cognitions Inventory (PTCI): The PTCI is a 33-item self-report questionnaire which assesses negative cognitions regarding a traumatic event and/or its sequelae. Items are framed as negative statements and agreement rated on a seven point Likert scale from 1 (totally disagree) to 7 (totally agree), with higher ratings indicating greater negative belief. A total score (PTCI-Total) can be derived as can three subscales (PTCI-Self, PTCI-World, and PTCI-Blame). PTCI-Self consists of 21-items that measure negative cognitions relating to the self (for example, 'if I think about the event, I will not be able to handle it'). PTCI-World consists of seven-items that measure negative cognitions relating to the world (for example, 'the world is a dangerous place'). PTCI-Blame consists of five items that measure negative cognitions relating to self-blame (for example, 'somebody else would not have gotten into this situation'). The PTCI has been used widely and has been shown to have acceptable psychometric properties (Beck et al., 2004).

\subsection{Plan for Analysis:}

A series of multiple regressions were planned with the PDS subscales as dependent variables and the PTCI subscales as independent predictors. In order to identify whether the PTCI subscales fit best with the DSM-5 avoidance symptoms versus the detachment and numbing symptoms we separated 
the PDS Avoidance and Numbing subscale into two: one to represent the avoidance symptoms (two items); and the other to represent the detachment and negative affect symptoms (five items).

\section{Results:}

Mean scores (standard deviations in parentheses) for PDS Intrusions $=6.35$ (4.23); PDS Avoidance $=$ 2.98 (2.17); PDS Numbing/Detachment = 6.33 (4.72); PDS Hyperarousal = 7.47 (4.65); PTCI Self $=$ 67.53 (35.43); PTCI World $=32.39$ (11.55) and PTCI Self Blame $=15.29$ (8.66). All measures showed good levels of internal consistency: PDS Intrusion $\alpha=.88$; PDS Avoidance $\alpha=.79$; PDS Numbing/Detachment $\alpha=.85$; PDS Hyperarousal $\alpha=.86$; PTCI Self $\alpha=.97$; PTCI World $\alpha=.92$; PTCI Blame $\alpha=.85$.

Bivariate correlations indicated statistically significant relationships between all subscales. In relation to how the PTCI subscales relate to the PDS subscales the largest associations were between: PTCI Self and PDS Numbing/Detachment $(r=.71, \mathrm{p}<.001)$; PTCI Self and PDS Hyperarousal $(\mathrm{r}=$ $.64, \mathrm{p}<.001)$; PTCI World and PDS Hyperarousal $(\mathrm{r}=.60, \mathrm{p}<.001)$ and PTCI World and PDS Numbing/Detachment $(\mathrm{r}=.56, \mathrm{p}<.001)$.

\subsection{Multiple Regressions:}

A series of regression analyses was conducted to explore the relative contribution of the three PTCI subscales in the prediction of individual PDS subscales (N.B. we use the word "predict" in the sense that it is used in regression analysis, to predict scores on one variable from known scores on other variables. It is not intended to imply causal association) (see Table One). 


\section{$\underline{\text { Table } 1 \text { about here }}$}

The PTCI accounted for $51.5 \%$ of the variance in Numbing/Detachment with all three subscales making unique contributions. The PTCI also accounted for $48.3 \%$ of the variance in Hyperarousal although only PTCI-self and PTCI-world were uniquely predictive. Only $34.1 \%$ of the variance in Avoidance was accounted for by the PTCI, again with both PTCI-self and PTCI-world making unique contributions, while the PTCI accounted for $30.9 \%$ of the variance in Intrusions with only PTCI-self being predictive.

\section{Discussion:}

The present study examined the predictive value of negative posttraumatic cognitions in relation to DSM-5 PTSD symptom clusters, in a large online sample of people reporting a reasonably diverse range of traumas. It is clear from the multiple regression analyses above that the PTCI subscales overlap to a greater extent with the PDS Numbing/Detachment subscale than any other, although we note the Hyperarousal subscale was nearly as strongly related. This is not surprising since the Numbing/Detachment and Hyperarousal subscales were highly correlated $(r=.74, p<.001)$. Other research suggests that hyperarousal is the strongest predictor of numbing (Litz, Schlenger, Weathers, Caddell, Fairbank, \& LaVange, 1997). These authors suggest it may be due to hyperarousal causing depletion of cognitive and emotional resources (leading to numbing) or that attempts at emotional suppression might lead to a rebound in attention. Nevertheless, the fact that PTCI subscales account for more of the variance, and that all three subscales make a unique contribution only to this subscale, it suggests these negative cognitions have most in common with Numbing/Detachment. 
Negative cognitions regarding the Self was the strongest independent predictor across the analyses which is consistent with earlier studies (Dorfel et al., 2008; Hatcher et al., 2009; Karl et al., 2009) that have considered the roles of appraisals in PTSD. However much of this previous work featured participants who had experienced road traffic accidents. Our results may therefore help generalise this finding to survivors of other types of traumatic event. Negative cognitions about the World were also a significant predictor across symptom clusters (barring the Intrusion cluster), yet appraisals related to Self-Blame only predicted Numbing/Detachment, further fuelling debate regarding their role in maintaining PTSD (Dorfel et al, 2008; Hatcher et al., 2009). It is also noteworthy, that even when statistically significant, the amount of variance contributed by PTCI Self-Blame was negligible and likely to be due to a Type I error given the very large sample size.

For clinicians, the results of our study imply that attention to posttraumatic cognitive content remains important. Negative meaning-making, related to a trauma or its consequences, seems most associated with numbing and emotional and social constriction. Knowledge of this may be particularly useful during the formulation and psychoeducation phases of treatment. As such, a rationale for active engagement with negative trauma related attributions can be encouraged (i.e. they are clinically important to attend to), paving the way for specific cognitive and behavioural interventions designed to reduce avoidance of distressing material, turn towards suffering (e.g. Lee, 2009), and re-engage with one's social surroundings. Further implications may be that attributions concerning oneself and the world may be the first to explore, while also probing for blame-related beliefs (which can remain highly problematic for an individual) as part of an overall clinical assessment. 
In terms of limitations, our study employed a cross-sectional design and so we cannot draw causal inferences regarding the relationship between negative posttraumatic cognitions and PTSD. Our use of an online survey methodology also means that our sample could suffer from a degree of selection bias. If anything, it likely contains a disproportionally greater number of higher level of socioeconomic status participants. However, in spite of these limitations there are also a number of strengths to note. Principally our sample size is large, aiding statistical power. In addition, while not necessarily representative of all those who experience trauma, it is also likely that our results will generalise to a wide range of sufferers by way of demographic factors (such as gender, age and ethnicity) as well as to clinical presentations given the variety of trauma experiences and range of symptoms reported.

To complement the current findings it will be important for future research to explore relationships between PTSD symptoms as they unfold over time. This would enable researchers to make inferences regarding causality and consider the importance of particular aspects of posttraumatic experience. Such greater specificity could aid model development and inform clinical practice. In this way a more comprehensive account of factors that might influence trauma related symptom presentations can be developed and existing models of PTSD more rigorously assessed.

In conclusion, our findings tentatively support the inclusion of posttraumatic appraisals within the Numbing/Detachment items of the DSM-5. However, the overlap with Hyperarousal needs to be explored further. 


\section{References:}

American psychiatric Association (1994). Diagnostic and statistical manual of mental disorders Fourth Edition. Washington DC: American Psychiatric Association.

American psychiatric Association (2013). Diagnostic and statistical manual of mental disordersFifth Edition. Washington DC: American Psychiatric Association.

Beck, J.G., Coffey, S.F., Palyo, S.A., Gudmundsdottir, B., Miller, L.M. \& Colder, C.R. (2004). Psychometric properties of the Posttraumatic Cognitions Inventory (PTCI): a replication with motor vehicle accident survivors. Psychological Assessment, 16(3), 289-298.

Buchanan \& Smith (1999) Using the Internet for psychological research: Personality testing on the World Wide Web. British Journal of Psychology, 90, 125-144.

Carek, V., Norman, P. \& Barton, J. (2010). Cognitive appraisals and posttraumatic stress disorder symptoms in informal caregivers of stroke survivors. Rehabilitation Psychology, 55(1), 91-96.

Dorfel, D., Rabe, S. \& Karl, A. (2008). Coping strategies in daily life as protective and risk factors for posttraumatic stress in motor vehicle accident survivors. Journal of Loss and Trauma, 13(5), 422440.

Ehlers, A. \& Clark, D.M. (2000). A cognitive model of posttraumatic stress disorder. Behaviour, Research and Therapy, 38, $319-345$.

Ehlers , A., Mayou, R.A. \& Bryant, B. (1998). Predictors of chronic PTD after road traffic accidents. Journal of Abnormal Psychology, 107, 508 - 519. 
Ehring, T., Ehlers, A. \& Glucksman, E. (2006). Contribution of cognitive factors to the prediction of post-traumatic stress disorder, phobia and depression after motor vehicle accidents. Behaviour Research and Therapy, 44(12), 1699-1716

Foa, E. B. (1995) Posttraumatic Diagnostic Scale (PDS). Microtest Q Assessment System. National Computer Systems Inc.

Foa, E. B., Cashman, L., Jaycox, L., \& Perry, K. (1997) The validation of a self-report measure of posttraumatic stress disorder: The Posttraumatic Diagnostic Scale. Psychological Assessment, 9, 445451.

Foa, E. B., Ehlers, A., Clark, D. M., Tolin, D. F., \& Orsillo, S. M. (1999) The Posttraumatic Cognitions Inventory (PTCI): Development and Validation. Psychological Assessment, 11, 303-314.

Friedman, M. J., Resick, P. A., Bryant, R. A., \& Brewin, C. R. (2011). "Considering PTSD for DSM5". Depression and Anxiety, 28 (9): 750-769. doi:10.1002/da.20767

Hatcher, M. B., Whitaker, C. \& Karl, A (2009) What predicts post-traumatic stress following spinal cord injury. British Journal of Health Psychology, 14, 3, 541-561. DOI:

$10.1348 / 135910708 \times 373445$

Karl, A., Rabe, S., Zollner, T., Maercker, A. \& Stopa, L. (2009) Negative self-appraisals in treatment-seeking survivors of motor vehicle accidents. Journal of Anxiety Disorders, 23(6):775-81. doi: 10.1016/j.janxdis.2009.03.001.

Lee, D.A. (2009). Compassion focused therapy to overcome shame and relentless self attack. In D. Sookman,\& R. Leahy (Eds), Treatment resistant anxiety disorders. New York: Routledge.

Litz, B., Schlenger, W., Weathers, F., Caddell, J., Fairbank, J., \& LaVange, L. (1997). Predictors of emotional numbing in posttraumatic stress disorder. Journal of Traumatic Stress, 10, 607-618. 
National Institute for Clinical Excellence (2005). Posttraumatic Stress Disorder (PTSD): The management of PTSD in adults and children in primary and secondary care. Retrieved 01.06.2010 from: http://www.nice.org.uk/CG26

Smith, M. A. \& Leigh, B. (1997) Virtual subjects: Using the Internet as an alternative source of subjects and research environment. Behaviour Research Methods, Instruments \& Computers, 29, 496-505. 
Table 1: PDS Intrusions, Avoidance, Numbing/Detachment and Hyperarousal regressed onto PTCI subscales

\begin{tabular}{|c|c|c|c|c|c|c|c|c|c|c|c|c|c|c|c|c|}
\hline \multirow[t]{2}{*}{ Model } & \multicolumn{4}{|c|}{ PDS Intrusion } & \multicolumn{4}{|c|}{ PDS Avoidance } & \multicolumn{4}{|c|}{ PDS Numbing } & \multicolumn{4}{|c|}{ PDS Hyperarousal } \\
\hline & B & $\begin{array}{l}\text { Std } \\
\text { Error }\end{array}$ & $\beta$ & $\mathrm{p}$ & B & $\begin{array}{l}\text { Std } \\
\text { Error }\end{array}$ & $\beta$ & $\mathrm{p}$ & B & $\begin{array}{l}\text { Std } \\
\text { Error }\end{array}$ & $\beta$ & $\mathrm{p}$ & B & $\begin{array}{l}\text { Std } \\
\text { Error }\end{array}$ & $\beta$ & $\mathrm{p}$ \\
\hline PTCI Self & .062 & .007 & .517 & .001 & .028 & .004 & .462 & .001 & .092 & .007 & .693 & .001 & .070 & .007 & .537 & .001 \\
\hline $\begin{array}{l}\text { PTCI } \\
\text { Blame }\end{array}$ & -.015 & .022 & -.031 & .498 & -.002 & .011 & -.009 & .846 & -.054 & .021 & -.099 & .010 & -.040 & .021 & -.075 & .055 \\
\hline $\mathrm{R}^{2}$ & .309 & & & & .341 & & & & .515 & & & & .483 & & & \\
\hline
\end{tabular}

Note: Subscale scores for the PDS were calculated for DSM-5 symptom clusters on the following basis: Intrusion (PDS items 22, 23, 24, 25, 26); Avoidance (PDS items 27, 28); Numbing/Detachment (PDS items 29, 30, 31, 32, 33) and Hyperarousal (PDS items 34, 35, 36, 37, 38). The earlier DSM-IV symptom clusters were identical except that DSM-IV Avoidance included all the items from DSM-5 Avoidance and DSM-5 Numbing/Detachment 
